\title{
Improving Video Quality in Network Paths With Bursty Losses
}

\author{
Felix Espina, Daniel Morato, Mikel Izal and Eduardo Magaña \\ Public University of Navarre \\ Campus de Arrosadía s/n, E-31006 Pamplona, Spain \\ Email: \{felix.espina, daniel.morato, mikel.izal, eduardo.magana\}@unavarra.es
}

\begin{abstract}
This paper presents a method to improve video quality at the receiver side of a streaming service when the network path presents losses in bursts. Technologies that aggregate packets into larger switched data units are typical scenarios for this kind of loss behavior. Optical Burst Switched (OBS) networks and Optical Packet Switched (OPS) networks are examples of such technologies. Video quality is evaluated by means of a no reference video quality metric (the Frame Starvation Ratio). Results show that using video frame classification at the edge node and service differentiation at the network, the playback quality can be substantially improved.
\end{abstract}

\section{INTRODUCTION}

Video for streaming services is nowadays digitalized using mainly coders from the MPEG family or proprietary (but similar in concept) ones [1]. MPEG2 and MPEG4/H.264 are the preferred solutions for digital compressed video.

In the MPEG standard, the decoding process for some video frames depends on previously decoded frames, so the drop of one frame has serious consequences on other frames [2]. This dependence among frames allows for a reduction on frame size keeping the image quality, due to similarities among frames close in time. However, as the frame size varies, the corresponding bit rate is variable. Thus, MPEG video typically leads to variable-bit-rate (VBR) traffic.

Video flows in a streaming service are assumed to be uni-directional UDP-like transport layer flows from a server farm. Due to the VBR nature of the video, the need for high bandwidth (especially with high definition video) and the growing demand of video contents, the server farm needs a high speed optical core network to distribute videos to remote users. In the short to medium term, Optical Burst Switching (a.k.a. OBS) [3] is an optical technology suitable for this task.

In an OBS network the ingress node aggregates packets into bursts. The bursts use an all-optical data plane to the egress node where they are disassembled into their contained packets. Bursts dropped by the network contain a sequence of packets resulting in bursty packet losses.

The prevalent burst formation mechanisms (a.k.a. burstifiers) in the literature are timer-based, size-based or a mixture of both timer- and size- based [4][5]. In a timer-based burstifier a timer of value $T_{\text {out }}$ is started on the arrival of a packet to an empty burst formation queue (one for each Forwarding Equivalence Class or FEC). When the timer expires, the burst is scheduled for transmission on the output port. The timer- based burstifier is the most frequently used in the literature as it can easily guarantee a maximum delay for the traffic.

Optical buffering in the core nodes can be implemented using Fiber Delay Lines, but usually it is nonexistent or scarce. Therefore, burst losses in the core network will be the result of output port contention that could not be solved by the core node. In this paper, the optical core network is represented as a black box as shown in Fig. 1, modeling the effect of interfering traffic to the video flow using burst loss ratio $p$ (independent losses). This is a common assumption as the losses in the OBS core network are less correlated than in a packet switched network due to its bufferless nature [6]. The burst loss ratio will be a network design parameter.

A dropped burst will contain packets from one or more video frames. Video servers usually send all packets from a frame back-to-back, so all these packets arrive at the burstifier approximately at the same time. It is assumed that if a packet from a frame enters into a burst created by a timer-based burstifier, then the rest of the packets from the frame enter too. Therefore, using a timer-based burstifier bursts will contain one or more whole frames.

For video visualization, the number of frames that could not be decoded due to losses and inter-frame dependencies is a more representative quality metric than the number of lost frames in the network. The number of lost frames is a Quality of Service $(Q o S)$ metric related to the network. The number of frames that could not be decoded is a Quality of Experience $(Q o E)$ metric related to the network and the subjective perception of quality of video on the user side.

The ingress node creates at least one burstifier per egress node. This paper shows that if the network can provide different loss ratios for bursts from different burstifiers a better result in non-decodable frames can be obtained even at the expense of more losses in the network. For this purpose, the OBS core network must implement QoS differentiation. It can be a relative QoS differentiation like the probabilistic preemption-based mechanism proposed in [7] or an absolute QoS differentiation like the early dropping and wavelength grouping proposed in [8] or the probabilistic preemption-based framework in [9]. With relative QoS, the burst loss ratios for the different classes (burst types) will maintain a preset and tunable ratio between them, but a maximum value for each class will not be guaranteed. With absolute QoS, the burst loss ratios for each individual class will not exceed a preset 


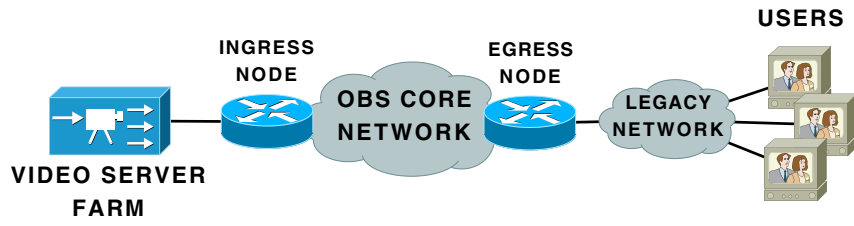

Fig. 1. General network scenario

and tunable value, different for each class.

In a reference scenario without differentiation, the ingress node will have one burstifier per video. In the scenario with differentiation, the ingress node will have more than one burstifier per video and different QoS strategies will be applied in the core OBS network. In this paper two scenarios will be studied. This paper will study this two scenarios, as this will allow to tune the video transport network for an objective quality user experience.

The rest of the paper is organized as follows. Section II introduces some important parameters of encoded video. Sections III and IV present the analytical study of the two proposed scenarios. Section V presents the validation of these analytical results. Finally, section VI concludes de paper.

\section{VIDEO CODIFICATION}

The MPEG standard defines three types of video frames [10]: intra-coded frames (I-frames), inter-coded or predicted frames (P-frames) and bidirectional coded frames (B-frames). I-frames can be decoded on their own. P-frames depend on the previous I- or P-frame and B-frames depend on the previous Ior P-frame and the following one of either type. The number of frames per second (frame-rate) is constant, and its inverse is the (constant) inter-frame time $T_{i f}$.

I-, P- and B-frames are grouped into GoPs (Groups of Pictures). A GoP is a sequence of frames beginning with an I-frame up to the frame before the next I-frame. The GoP structure is the pattern of I-, P- and B-frames used inside every GoP. A regular GoP structure is usually described as $G x B y$ where $x$ is the number of frames in the GoP and $y$ is the number of contiguous B-frames. For example, the GoP structure could be $G 12 B 2$ or IBBPBBPBBPBB. Different GoP sizes and structures, even changes of the GoP structure in a stream, are possible. However, most videos are coded using only one GoP structure.

A GoP structure like IBBPBBPBBPBB is shown in presentation order, the order in which the frames will be shown to the user. However, as B-frames need the previous and the following I- or P-frame in order to be coded/decoded, the coding/decoding order will be different. The coding/decoding order will be $I b b P B B P B B P B B i B B$ for a $G 12 B 2 \mathrm{GoP}$ structure, where the frames in lower-case correspond to frames from the previous or the next GoP. The last example is an open GoP. In an open GoP the last B-frames depend on the I-frame from the next GoP. In a closed GoP there is no dependence with frames out of the GoP. A closed GoP ends with a Pframe in presentation order, like for example in $G 9 B 3$ or
$I B B B P B B B P$. The transmission order of frames is usually the coding/decoding order. This ordering decides which frames are lost when a burst is dropped. In this paper the structure of a GOP will be always considered in coding/decoding order, not in presentation order.

The drop of one frame has serious consequences on other frames. As the MPEG standard does not define a specific coder/decoder behavior, a worst case scenario is assumed in this paper, where the loss of one frame will prevent decoding any other frame that depends on it.

As an example, Fig. 2 shows, on the left side, a frame that has been dropped in the network. In the right side of the same figure, all the frames that could not be decoded due to that loss are marked with dashed lines. The loss of a P-frame triggers the impossibility to decode all the following frames in the $\mathrm{GoP}$ and the B-frames previous to it and after another I- or P-frame.

A new burstifier management method is proposed in section IV, where the different types of video frames are aggregated using different burstifiers.

The Frame Loss Ratio $(F L R)$ is defined as $F_{l} / F$, where $F_{l}$ is the number of lost frames in the network and $F$ is the total number of frames. The Frame Starvation Ratio (FSR) is defined as $F_{n d} / F$, where $F_{n d}$ is the number of frames that could not be decoded due to losses and inter-frame dependencies. It is clear that FSR $\geq F L R$. FSR is a no reference (NR) metric [11], as no information from the original video is needed in order to compute its value. Error Correction schemes implemented by the decoder will reduce the FSR and improve the QoE at the user end, maintaining the FLR or QoS metric. However, applying Error Correction schemes is very specific on the decoder implementation and it can not be introduced in a general analytical model as proposed in this paper.

\section{SCENARIO WITHOUT DIFFERENTIATION}

The scenario where all the frames from a video flow use the same burstifier, regardless of frame type, was partially studied in [12]. An expression for FLR and FSR was obtained, but only an iterative and not formal way to compute $F S R$ was presented. In this paper, an algebraic and systematic way to compute $F S R$ is demonstrated.

Assuming a burst loss ratio $p$ at the core OBS network, bursts from different burstifiers do not interfere with each other. It was shown in [12] that $F L R=p$ and $F S R=\phi / F_{b} \cdot F L R$, where $F_{b}=\left\lceil T_{\text {out }} / T_{\text {if }}\right\rceil$ is the number

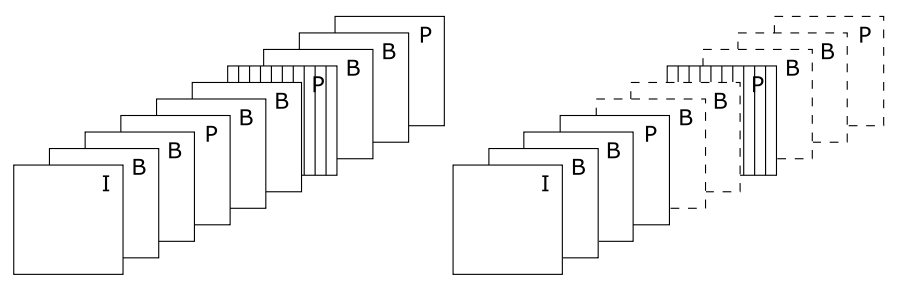

Fig. 2. Example of inter-frame dependence in a GoP 
of frames per burst and $\phi$ (labeled as $E\left[X_{F_{b}}\right]$ in [12]) is the average number of frames that can not be decoded due to the loss of $F_{b}$ consecutive frames. Remember that $T_{\text {out }}$ is the burst formation timer value and $T_{i f}$ is the inter-frame time. On the following, $\phi$ is directly obtained (1), without iterative computation methodologies as those presented in [12].

$$
\phi=\frac{1}{x} \sum_{j=1}^{x} \phi[j]
$$

$\phi[j]$ is defined as the number of frames that could not be decoded due to losses and inter-frame dependencies when a burst of $F_{b}$ frames is lost where the first frame in the burst is the $j^{\text {th }}$ frame of the GoP. As the GoP structure $G x B y$ is very different from open to closed GoPs, $\phi[j]$ will vary.

A GoP has only one I-frame and it is always the first frame $(j=1)$. We define $\phi_{\mathrm{I}}\left[T_{\text {out }}\right]$ as $\phi[j=1]$. The loss of a burst started by an I-frame will cause all the GoP to not be decoded and, in the case of open GoPs, even the last B-frames block from the previous GoP will be useless. Equation (2) provide $\phi_{\mathrm{I}}\left[T_{\text {out }}\right]$ for open and closed GoPs.

$$
\phi_{\mathrm{I}}\left[T_{\text {out }}\right]= \begin{cases}x\left\lceil\frac{T_{\text {out }}}{x T_{\text {if }}}\right]+y & \text { open GoP } \\ x\left\lceil\frac{T_{\text {out }}}{x T_{\text {if }}}\right\rceil & \text { closed GoP }\end{cases}
$$

A GoP has $G_{\mathrm{P}}=\lfloor(x-1) /(y+1)\rfloor \mathrm{P}$-frames inside because there has to be as many $\mathrm{P}$-frames as complete $\mathrm{B}$ frames blocks in the GoP. The loss of a P-frame always makes impossible to decode all the following frames in the GoP. So, the number of frames that could not been decoded when a burst started by a P-frame is lost will depend on which P-frame is lost. In an open GoP, the Pframes are the frames at position $j=1+i(y+1) ; i=1 \ldots G_{\mathrm{P}}$ and in a closed GoP, P-frames are the frames at position $j=2+(i-1)(y+1)=1+i(y+1)-y ; i=1 \ldots G_{\mathrm{P}}$. If the $\mathrm{i}^{\text {th }} \mathrm{P}$-frame is lost it will always cause that $x+y-i(y+1)$ frames will not been decoded. If $T_{\text {out }}$ is large enough to reach the I-frame from the next GoP, then all frames from the next GoP will not be decoded.

Equation (3) provides $\phi[j]$ for the case of a loss starting by a P-frame. It is computed as $\phi_{\mathrm{P}}\left[i ; T_{\text {out }}\right]$ where $i=1 \ldots G_{\mathrm{P}}$, $j=1+i(y+1)$ for an open GoP and $j=1+i(y+1)-y$ for a closed one.

$$
\phi_{\mathrm{P}}\left[i ; T_{\text {out }}\right]=\left\lceil\frac{T_{\text {out }}+(j-1) T_{i f}}{x T_{i f}}\right\rceil x+y-i(y+1)
$$

In an open GoP, the B-frames are the frames at position $j=1+i(y+1)+k ; i=0 \ldots G_{\mathrm{P}}$ and in a closed GoP they are at position $j=1+i(y+1)-y+k ; i=1 \ldots G_{\mathrm{P}}$. In both cases $k=1 \ldots y$. The loss of a B-frame does not affect any other frame. As the timer $T_{\text {out }}$ gets larger, more frames get inside the lost burst whose first frame is a B-frame. Eventually, an I-frame (if the frames are from the last B-frames block) or a P-frame (otherwise) will be lost too. This will cause that other not dropped frames will not been decoded. For the $\mathrm{k}^{\text {th }} \mathrm{B}$-frame from a B-frames block, this happens when $T_{\text {out }}>(y-k+1) T_{i f}$.

Equation (4) provides $\phi[j]$ where $T_{B}[k]=(y-k+1) T_{i f}$, $T^{\prime}=T_{\text {out }}+(j-1) T_{i f}-x T_{i f}$ and $T^{\prime \prime}=T_{\text {out }}-T_{B}[k]$. Obviously, $\phi_{\mathrm{I}}\left[T^{\prime}\right]$ and $\phi_{\mathrm{P}}\left[i+1 ; T^{\prime \prime}\right]$ use the different expressions for open and closed GoPs introduced earlier.

$$
\phi[j]= \begin{cases}\left\lceil\frac{T_{\text {out }}}{T_{\text {if }}}\right\rceil & \text { if } T_{\text {out }} \leq T_{B}[k] \\ \left\lfloor\frac{i}{G_{\mathrm{P}}}\right\rfloor \phi_{\mathrm{I}}\left[T^{\prime}\right]+y-k+1+ & \\ +\left(1-\left\lfloor\frac{i}{G_{\mathrm{P}}}\right\rfloor\right) \phi_{\mathrm{P}}\left[i+1 ; T^{\prime \prime}\right] & \text { if } T_{\text {out }}>T_{B}[k]\end{cases}
$$

Table I shows the values of $\phi$ for several GoP structures and different $F_{b}$.

\section{SCENARIO WITH DIFFERENTIATION}

In this section, the results from the classification of different frame types into separate burstifiers is evaluated analytically. Fig. 3 shows this classification where each burstifier collects frames from a single video frame type, creating I-bursts (bursts containing only I-frames), P-bursts or B-bursts. As far as we know, this method is an important contribution from this paper.

Frame type differentiation allows different loss ratios per frame type and to obtain better quality results. As side effects, burst size variability will get reduced and the total number of bursts could increase. The first one has a positive impact on the scheduler performance at the core switching nodes of the network. The second one could have a negative impact on the network and it must be studied further.

A QoS scheme capable of providing different loss rates for these flows in the network is assumed. Let $p^{\mathrm{I}}, p^{\mathrm{P}}$ and $p^{\mathrm{B}}$ be the loss ratios in the network for the three flows from a streaming movie. We use $p^{\tau}$ where $\tau \in\{\mathrm{I}, \mathrm{P}, \mathrm{B}\}$ to designate any of the burst loss ratios.

TABLE I

AVERAGE LOSSES DUE TO INTER-FRAME DEPENDENCE, $\phi$

\begin{tabular}{c|ccc}
$F_{b}$ & $\mathrm{G} 12 \mathrm{~B} 2$ & $\mathrm{G} 16 \mathrm{~B} 7$ & $\mathrm{G} 16 \mathrm{~B} 14$ \\
\hline 1 & 3.83 & 3.25 & 2.81 \\
2 & 7.33 & 6.37 & 4.62 \\
3 & 10.5 & 9.37 & 6.37 \\
4 & 11.5 & 12.25 & 8.06 \\
5 & 12.5 & 15 & 9.68 \\
6 & 13.5 & 17.62 & 11.25 \\
7 & 14.5 & 20.12 & 12.75 \\
8 & 15.5 & 22.5 & 14.18 \\
9 & 16.5 & 23.5 & 15.56 \\
10 & 17.5 & 24.5 & 16.87
\end{tabular}

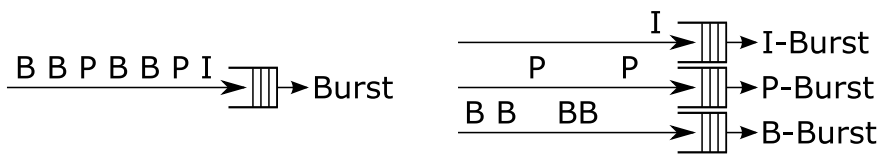

Fig. 3. All frames in the same burstifier (left) or in different burstifiers (right) 
The average number of dropped $\tau$-bursts is $b_{l}^{\tau}=b^{\tau} p^{\tau}$, where $b^{\tau}$ is the number of $\tau$-bursts that a video generates when a burstifier with timer value $T_{\text {out }}$ is used. The total number of $\tau$-frames in the video is $F^{\tau}=F_{b}^{\tau} b^{\tau}$, where $F_{b}^{\tau}$ is the number of $\tau$-frames inside each $\tau$-burst. Let $G_{\tau}$ be the number of $\tau$-frames inside a GoP $\left(x=G_{\mathrm{I}}+G_{\mathrm{P}}+G_{\mathrm{B}}\right)$ and $M$ the total number of GoPs in a video. Then $F^{\tau}=G_{\tau} M$. The number of dropped $\tau$-frames in the network from a video is $F_{l}^{\tau}=F_{b}^{\tau} b_{l}^{\tau}=F_{b}^{\tau} b^{\tau} p^{\tau}=F^{\tau} p^{\tau}$. Equation (5) computes the FLR.

$$
\begin{aligned}
F L R= & \frac{F_{l}}{F}=\frac{\sum_{\tau \in\{I, P, B\}} F_{l}^{\tau}}{\sum_{\tau \in\{I, P, B\}} F^{\tau}}=\frac{\sum_{\tau \in\{I, P, B\}} F^{\tau} p^{\tau}}{\sum_{\tau \in\{I, P, B\}} F^{\tau}}= \\
= & \frac{\sum_{\tau \in\{I, P, B\}} M G_{\tau} p^{\tau}}{\sum_{\tau \in\{I, P, B\}} M G_{\tau}}=\frac{\sum_{\tau \in\{I, P, B\}} G_{\tau} p^{\tau}}{\sum_{\tau \in\{I, P, B\}} G_{\tau}}= \\
= & \frac{\sum_{\tau \in\{I, P, B\}} G_{\tau} p^{\tau}}{x}
\end{aligned}
$$

The FLR depends on the burst loss ratio $p^{\tau}$ for each type of bursts (a design parameter) and on the number of $\tau$-frames inside a GoP, that only depends on the GoP structure.

It is clear that each GoP structure $G x B y$ has only one Iframe, $G_{\mathrm{I}}=1$. The total number of P-frames inside a GoP is $G_{\mathrm{P}}=\lfloor(x-1) /(y+1)\rfloor$. The total number of B-frames inside a GoP is $G_{\mathrm{B}}=y\lceil(x-1) /(y+1)\rceil$, because there have to be $y$ B-frames for each B-frames block in the GoP.

$\phi^{\tau}$ is defined as the average number of frames that could not be decoded due to losses and inter-frame dependencies when a $\tau$-burst is lost. The number of frames from a video that could not be decoded due to losses of $\tau$-bursts and interframe dependencies is $F_{n d}^{\tau}$, and as in [12], it can approximated by $\phi^{\tau} b_{l}^{\tau}$. The $F S R$ is finally obtained using the algebra in (6).

$$
\begin{aligned}
F S R & =\frac{F_{n d}}{F}=\frac{\sum_{\tau \in\{I, P, B\}} F_{n d}^{\tau}}{\sum_{\tau \in\{I, P, B\}} F^{\tau}} \approx \frac{\sum_{\tau \in\{I, P, B\}} \phi^{\tau} b_{l}^{\tau}}{\sum_{\tau \in\{I, P, B\}} F^{\tau}}= \\
& =\frac{\sum_{\tau \in\{I, P, B\}} \phi^{\tau} b^{\tau} p^{\tau}}{\sum_{\tau \in\{I, P, B\}} F^{\tau}}=\frac{\sum_{\tau \in\{I, P, B\}} \phi^{\tau} \frac{G_{\tau} M}{F_{b}^{\tau}} p^{\tau}}{\sum_{\tau \in\{I, P, B\}} G_{\tau} M}= \\
& =\frac{\sum_{\tau \in\{I, P, B\}} \frac{\phi^{\tau}}{F_{b}^{\tau}} G_{\tau} p^{\tau}}{x}
\end{aligned}
$$

Therefore, FSR as FLR depend on $p^{\tau}$ and $G_{\tau}$, but also on $\phi^{\tau} / F_{b}^{\tau}$. The values of $G_{\tau}$ and $\phi^{\tau} / F_{b}^{\tau}$ are the result of the GoP structure used in the video coding process.

$\phi^{\mathrm{B}}$ is the average number of frames that could not be decoded due to losses and inter-frame dependencies when a $B$-burst (a burst containing only B-frames) is lost. B-frames are the only ones that do not affect other frames, so $\phi^{\mathrm{B}}=F_{b}^{\mathrm{B}}$.
$\phi^{\mathrm{I}}$ is the average number of frames that could not be decoded due to losses and inter-frame dependencies when an I-burst (a burst containing only I-frames) is lost. It is clear that the loss of an I-frame affects all the frames in the GoP, and on open GoPs it also affects to the last B-frames block in the previous GoP. So, for open GoPs $\phi^{\mathrm{I}}=(x+y) F_{b}^{\mathrm{I}}$ and for closed GoPs $\phi^{\mathrm{I}}=x F_{b}^{\mathrm{I}}$.

$\phi^{\mathrm{P}}$ is the average number of frames that could not be decoded due to losses and inter-frame dependencies when a P-burst (a burst containing only P-frames) is lost. $\mathrm{P}$ - and $\mathrm{B}$-frames depend on the correct decoding of the previous Pframes in the GoP. So, all the frames counting from the first P-frame in the burst to the end of the GoP for the last P-frame in the burst will be not be decoded. An exception are the Iframes from the affected GoPs as they do not depend on any other frame.

Obtaining an equation for $\phi^{\mathrm{P}}$ is not as simple as for $\phi^{\mathrm{I}}$ and $\phi^{\mathrm{B}}$ because it strongly depends on the GoPs structure and the relation between the burstifier timer $T_{\text {out }}$ and the video inter-frame time $T_{i f}$. These dependencies cause, for example, that with a $G 12 B 2 \mathrm{GoP}$ and $3 T_{\text {if }}<T_{\text {out }}<6 T_{\text {if }}$ only two different types of P-bursts will be created: one with the first two P-frames in the GoP and the second one with the last Pframe. If the video transmission would begin with the second P-frame in the GoP it would only create one burst starting with that P-frame and in the next GoP it would converge to one of the other two types (see Fig. 4).

Taking this into account, in order to compute $\phi^{\mathrm{P}}$ we define $\phi^{\mathrm{P}}[i]$ as the average number of frames that could not be decoded due to losses and inter-frame dependencies when a burst with only $P$-frames is lost, being the $i^{\text {th }} P$-frame from the GoP the first frame in the burst.

If $T_{\text {out }}$ is less than the GoP duration, then for open GoPs the loss of the $i^{\text {th }} \mathrm{P}$-frame will cause that $x+y-i(y+1)$ frames will not be decoded. For closed GoPs, this loss will cause that $x-1-(i-1)(y+1)=x+y-i(y+1)$ frames will not be decoded. If $T_{\text {out }}$ is large enough to reach the first P-frame from the next GoP, then all frames from the next GoP except the I-frame will not be decoded. This happens when $T_{\text {out }}>x T_{i f}-T^{P}[i]$, where $T^{P}[i]=(i-1)(y+1) T_{i f}$. So, for any GoP structure and any $T_{\text {out }},(7)$ computes $\phi^{\mathrm{P}}[i]$.

$$
\begin{aligned}
\phi^{\mathrm{P}}[i] & =\left(\left\lceil\frac{T_{\text {out }}+T^{P}[i]}{x T_{i f}}\right\rceil-1\right)(x-1)+x+y-i(y+1)= \\
& =\left\lceil\frac{T_{\text {out }}+T^{P}[i]}{x T_{\text {if }}}\right\rceil(x-1)-(i-1)(y+1)
\end{aligned}
$$

$\phi^{\mathrm{P}}$ is expressed in (8) as the weighted average of $\phi^{\mathrm{P}}[i]$, where $\delta[i]$ is 1 if the $i^{\text {th }} \mathrm{P}$-frame can be the first frame of

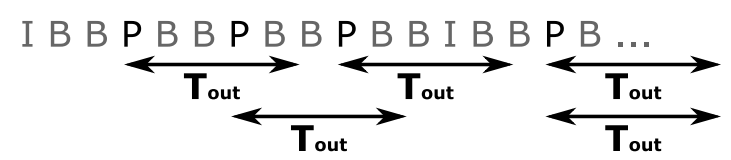

Fig. 4. P-bursts created with $3 T_{i f}<T_{\text {out }}<6 T_{i f}$ for a $G 12 B 2 \mathrm{GoP}$ 
a burst and 0 otherwise. Therefore, $\phi^{\mathrm{P}}$ depends on the GoP structure and the relationship between $T_{\text {out }}$ and $T_{i f}$.

$$
\phi^{\mathrm{P}}=\frac{\sum_{i=1}^{G_{\mathrm{P}}} \delta[i] \phi^{\mathrm{P}}[i]}{\sum_{i=1}^{G_{\mathrm{P}}} \delta[i]}
$$

We define $F_{b}^{\mathrm{P}}[i]$ as the average number of $P$-frames inside a P-burst, being the $i^{\text {th }} P$-frame from the GoP the first frame in the burst. If $T_{\text {out }} \leq\left(G_{P}+1-i\right)(y+1) T_{i f}=$ $G_{P}(y+1) T_{i f}-T^{P}[i]$, then the P-burst has only Pframes from the same GoP. If $T_{\text {out }}>G_{P}(y+1) T_{i f}-$ $T^{P}[i]$, then some P-frames from the next GoP are lost. So, for any GoP and any $T_{\text {out }}$, (9) computes $F_{b}^{\mathrm{P}}[i]$, where $T^{\prime \prime \prime}=T_{\text {out }}-\left\lfloor T_{\text {out }} /\left(x T_{\text {if }}\right)\right\rfloor x T_{\text {if }}$ and $\alpha=y+1$ for open GoPs and $\alpha=1$ for closed GoPs.

$$
F_{b}^{\mathrm{P}}[i]= \begin{cases}G_{P}\left\lfloor\frac{T_{o u t}}{x T_{i f}}\right\rfloor+\left\lceil\frac{T^{\prime \prime \prime}}{(y+1) T_{i f}}\right\rceil & \text { if } \frac{T^{\prime \prime \prime}+T^{P}[i]}{(y+1) T_{i f}} \leq G_{P} \\ G_{P}\left\lfloor\frac{T_{o u t}}{x T_{i f}}\right\rfloor+\left\lceil\frac{T^{\prime \prime \prime}-\alpha T_{i f}}{(y+1) T_{i f}}\right\rceil & \text { if } \frac{T^{\prime \prime \prime}+T^{P}[i]}{(y+1) T_{i f}}>G_{P}\end{cases}
$$

$F_{b}^{\mathrm{P}}$ is expressed in (10) as the weighted average of $F_{b}^{\mathrm{P}}[i]$.

$$
F_{b}^{\mathrm{P}}=\frac{\sum_{i=1}^{G_{\mathrm{P}}} \delta[i] F_{b}^{\mathrm{P}}[i]}{\sum_{i=1}^{G_{\mathrm{P}}} \delta[i]}
$$

Substituting the previous results into (5) and (6) we obtain the FLR and FSR as shown in (11) and (12), where $\phi^{\mathrm{I}} / F_{b}^{\mathrm{I}}$ is $x+y$ for open GoPs and $x$ for closed GoPs, and $\phi^{\mathrm{P}} / F_{b}^{\mathrm{P}}$ is obtained from (8) and (10).

$$
\begin{aligned}
F L R & =\frac{p^{\mathrm{I}}+\left\lfloor\frac{x-1}{y+1}\right\rfloor p^{\mathrm{P}}+y\left\lceil\frac{x-1}{y+1}\right\rceil p^{\mathrm{B}}}{x} \\
F S R & =\frac{\frac{\phi^{\mathrm{I}}}{F_{b}^{\mathrm{I}}} p^{\mathrm{I}}+\frac{\phi^{\mathrm{P}}}{F_{b}^{\mathrm{P}}}\left\lfloor\frac{x-1}{y+1}\right\rfloor p^{\mathrm{P}}+y\left\lceil\frac{x-1}{y+1}\right\rceil p^{\mathrm{B}}}{x}= \\
& =\frac{\frac{\phi^{\mathrm{I}}}{F_{b}^{\mathrm{I}}} p^{\mathrm{I}}+\frac{\phi^{\mathrm{P}}}{F_{b}^{\mathrm{P}}}\left\lfloor\frac{x-1}{y+1}\right\rfloor p^{\mathrm{P}}+y\left\lceil\frac{x-1}{y+1}\right\rceil p^{\mathrm{B}}}{p^{\mathrm{I}}+\left\lfloor\frac{x-1}{y+1}\right\rfloor p^{\mathrm{P}}+y\left\lceil\frac{x-1}{y+1}\right\rceil p^{\mathrm{B}}} F L R
\end{aligned}
$$

\section{RESUltS VALIDATION}

The expressions for FLR and FSR for the two scenarios were checked by simulation using traces of movie files from [13] that use different GoPs and frame-rates. Several different video traces and burst loss ratio $p$ were used in the simulations. Due to space constraints, only global results for the Lord of the Rings III (LOTR3) video trace and $p=0.01$ will be shown, although no significant difference was found with other combinations.
The simulator generates bursts from the video trace according to the burstification parameters. As stated before, the optical core network is represented as a black box modeling the effect of interfering traffic to the video flow as a burst loss ratio. The receiver node computes the $F L R$ and based on the arriving frames and the GoP structure it also calculates the $F S R$. For each iteration, the simulator randomly selects the first frame from the movie and it uses the video trace in a circular way until the whole movie has been transmitted.

Fig. 5 shows $F S R$ for the scenario without differentiation, for the movie trace file LOTR3 that used $G 12 B 2$ and $T_{i f}=40 \mathrm{~ms}$ and for Tokyo Olympics (TOK) that uses G16B7 and $T_{i f}=33.33 \mathrm{~ms}$. The $95 \%$ confidence intervals are also presented. The analytical results match quite well with the simulations for both videos. As the timer value $T_{\text {out }}$ grows, more frames are inside each burst. Frames that were not going to be decoded if the first frame of the burst were lost, are now inside the burst and are lost. This makes the FSR tend to the $F L R$ and therefore to the burst loss ratio $p$.

In order to obtain comparable results, the scenarios with differentiation use the same average burst loss ratio $p$ as the scenario without differentiation. That is, $\left(b_{l}^{\mathrm{I}}+b_{l}^{\mathrm{P}}+b_{l}^{\mathrm{B}}\right) /\left(b^{\mathrm{I}}+\right.$ $\left.b^{\mathrm{P}}+b^{\mathrm{B}}\right)=\left(b^{\mathrm{I}} p^{\mathrm{I}}+b^{\mathrm{P}} p^{\mathrm{P}}+b^{\mathrm{B}} p^{\mathrm{B}}\right) /\left(b^{\mathrm{I}}+b^{\mathrm{P}}+b^{\mathrm{B}}\right)=p$. This burst loss ratio can be achieved using different combinations of the $\tau$-burst loss ratios $p^{\tau}$. The studied combinations are:

- The three ratios $p^{\tau}$ are equal to the burst loss ratio $p$. This scenario is labeled in Fig. 6 and Fig. 7 as equal ratiosSim. for simulation values and equal ratios-Analytical for analytical results.

- I- and P-bursts are not dropped $\left(p^{\mathrm{I}}=p^{\mathrm{P}}=0\right)$ and all losses are from B-bursts. This is labeled as I,P no lossSim. for simulation values and I,P no loss-Analytical for analytical results in Fig. 6 and Fig. 7.

- I-bursts are not dropped $\left(p^{\mathrm{I}}=0\right)$ and losses are equally split between P- and B-bursts. Labeled as I no lossSim. for simulation values and I no loss-Analytical for analytical results in Fig. 6 and Fig. 7.

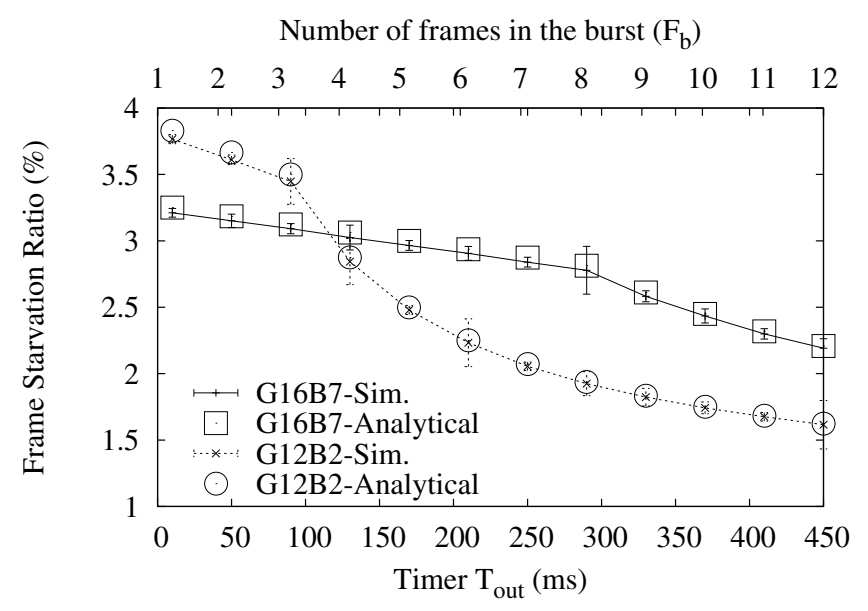

Fig. 5. Analytical and simulation comparison of FSR for different GoP structures and $p=10^{-2}$ 


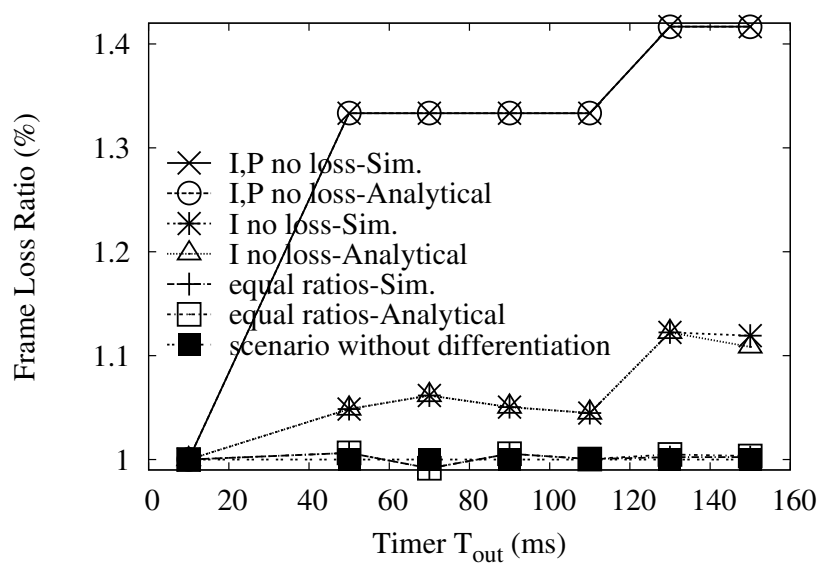

Fig. 6. Simulation and analytical results for FLR

Fig. 6 compares simulation and analytical results for $F L R$. The results verify the correctness of (11). In most scenarios, frame type separation into different burstifiers results in higher $F L R$ compared to the scenario where all frames get into the same burstifier. As the timer $T_{\text {out }}$ grows, more B-frames are aggregated into each burst and less B-bursts are created. When all the losses are only from B-bursts the result will be a higher $F L R$ (higher number of frames lost) than when the losses are shared among the three types of bursts. However, as the loss of B-frames does not affect the decoding process of other frames, the FSR can be reduced even with higher FLR.

Fig. 7 shows the FSR for the same scenarios. Analytical and simulation results match quite well. The $F S R$ values improve (get lower) for scenarios where the losses of I- or P-bursts are reduced. Keeping the same proportion of losses in the network, a higher priority (lower loss ratio) for I-bursts and P-bursts at the expense of higher loss ratio of B-bursts provides a better result in frames that could not be decoded. Therefore, the FSR video quality metric can be improved using frames differentiation.

\section{CONCLUSIONS}

This paper has shown how to compute the Frame Starvation Ratio for the case of a network where losses take place in bursts, like for example an OBS network. However, the results are general enough and apply to any technology with bursty losses or any technology that aggregates traffic into larger frames (for example some Optical Packet Switching architectures). Reducing the burst size to one packet, the results apply also to any packet switched technology.

Using frame type classification and aggregation at the edge of the network and providing different priorities in the end-toend path, the video quality at the receiver side can be improved even at the expense of higher network losses. The methodology described allows a network designer to select the video GoP structure and burstifier timeout value (or aggregation time for OPS or other technology) at the edge node that provides an objective quality result measured as the proportion of frames that were not shown to the user.

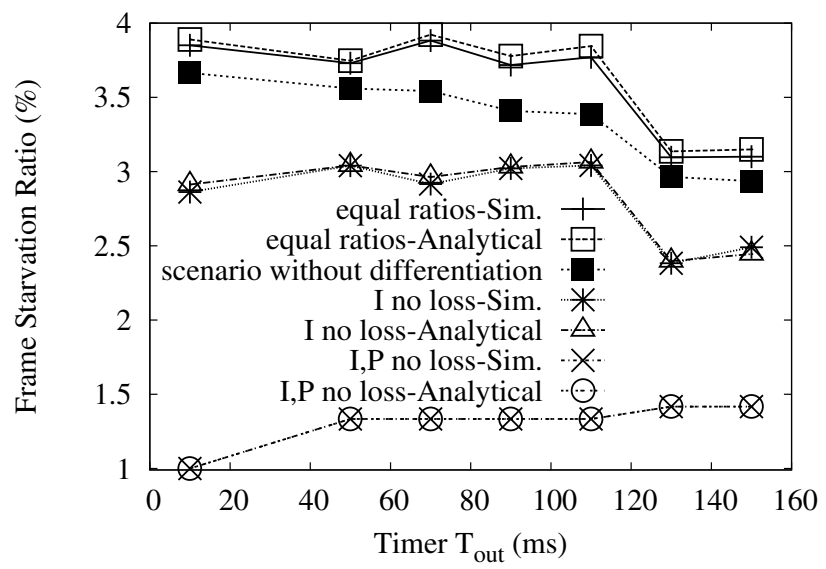

Fig. 7. Simulation and analytical results for FSR

\section{ACKNOWLEDGMENT}

This work was supported by the Spanish Ministry of Science and Innovation through the research project INSTINCT (TEC2010-21178-C02-01). The authors want to thank Spanish thematic network FIERRO (TEC2010-12250-E).

\section{REFERENCES}

[1] P. Seeling and M. Reisslein, "Evaluating multimedia networking mechanisms using video traces," Potentials, IEEE, vol. 24, no. 4, pp. 21-25, Oct.-Nov. 2005.

[2] O. A. Lotfallah, M. Reisslein, and S. Panchanathan, "A framework for advanced video traces: evaluating visual quality for video transmission over lossy networks," EURASIP J. Appl. Signal Process., 2006.

[3] C. Qiao and M. Yoo, "Optical burst switching (OBS) - a new paradigm for an optical internet," J. High Speed Netw., vol. 8, pp. 69-84, March 1999.

[4] J. Choi, J. Choi, and M. Kang, "Dimensioning burst assembly process in optical burst switching networks," IEICE Transactions on Communications, vol. E88-B, no. 10, pp. 3855-3863, October 2005.

[5] X. Yu, Y. Chen, and C. Qiao, "A study of traffic statistics of assembled burst traffic in optical burst switched networks," in Proceedings of SPIE Opticomm, July 2002, pp. 149-159.

[6] X. Yu, J. Li, X. Cao, Y. Chen, and C. Qiao, "Traffic statistics and performance evaluation in optical burst switched networks," IEEE Journal of Lightwave Technology, vol. 22, no. 12, pp. 2722- 2738, Dec. 2004.

[7] C. W. Tan, G. Mohan, and J.-S. Lui, "Achieving multi-class service differentiation in WDM optical burst switching networks: a probabilistic preemptive burst segmentation scheme," Selected Areas in Communications, IEEE Journal on, vol. 24, no. 12, pp. 106-119, Dec. 2006.

[8] Q. Zhang, V. Vokkarane, J. Jue, and B. Chen, "Absolute QoS differentiation in optical burst-switched networks," Selected Areas in Communications, IEEE Journal on, vol. 22, no. 9, pp. 1781-1795, Nov. 2004.

[9] M. H. Phung, K. C. Chua, G. Mohan, M. Motani, and T. C. Wong, "An absolute QoS framework for loss guarantees in optical burst-switched networks," Communications, IEEE Transactions on, vol. 55, no. 6, pp. 1191-1201, June 2007.

[10] D. Heyman and T. Lakshman, "Source models for VBR broadcast-video traffic," IEEE/ACM Transactions on Networking, vol. 4, no. 1, pp. 4048, February 1996.

[11] M. Fiedler, T. Hossfeld, and P. Tran-Gia, "A generic quantitative relationship between quality of experience and quality of service," Network, IEEE, vol. 24, no. 2, pp. 36-41, March-April 2010.

[12] F. Espina, D. Morato, M. Izal, and E. Magaña, "The effect of burst formation timers on video streaming over optical burst switched networks," in Proceedings of BROADNETS, September 2008.

[13] G. V. der Auwera, P. T. David, and M. Reisslein, "Traffic characteristis of H.264/AVC variable bit rate video," Arizona State University, Tech. Rep., March 2007. 